\title{
Simultaneous integrated coronary artery revascularization with long-term angiographic follow-up
}

Bob Kiaii, MD, FRCSC, ${ }^{a}$ R. Scott McClure, MD, ${ }^{\text {a }}$ Peter Stewart, MBBS, FRACP, ${ }^{\mathrm{b}}$ Reiza Rayman, MD, ${ }^{a}$ Stuart A. Swinamer, MD, ${ }^{a}$ Yoshihiro Suematsu, MD, ${ }^{a}$ Stephanie Fox, RRT, ${ }^{a}$ Jennifer Higgins, BSc, ${ }^{a}$ Caroline Albion, BSc, William J. Kostuk, MD, FRCPC, ${ }^{\text {b }}$ David Almond, MD, FRCPC, ${ }^{\text {b }}$ Kumar Sridhar, MD, FRCPC, ${ }^{\text {b }}$ Patrick Teefy, MD, FRCPC, George Jablonsky, MD, FRCPC, ${ }^{\text {b }}$ Pantelis Diamantouros, MD, FRCPC, ${ }^{b}$ Wojciech B. Dobkowski, MD, FRCPC, ${ }^{c}$ Philip Jones, MD, FRCPC, ${ }^{c}$ Daniel Bainbridge, MD, FRCPC, ${ }^{c}$ Ivan Iglesias, MD, ${ }^{c}$ John Murkin, MD, FRCPC, ${ }^{c}$ Davy Cheng, MD, FRCPC, ${ }^{c}$ and Richard J. Novick, MD, FRCSC, FACS ${ }^{a}$

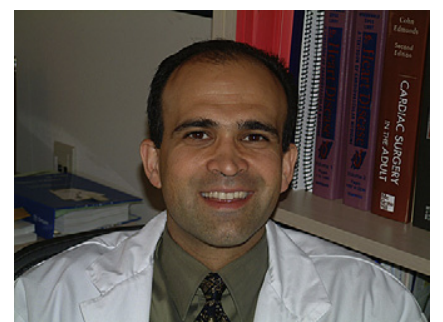

Dr Bob Kiaii
From the Departments of Surgery, Medicine, ${ }^{\mathrm{b}}$ and Anesthesia and Perioperative Medicine, ${ }^{c}$ University of Western Ontario, London Health Sciences Centre, University Hospital, Ivey Cardiac Centre, London, Ontario, Canada.

The construction of the infrastructure (hybrid operative suite with angiographic equipment) necessary to undergo the current case series was provided in the form of a grant from the Canada Foundation of Innovation.

Received for publication June 17, 2007; revisions received Jan 25, 2008; accepted for publication Feb 15, 2008.

Address for reprints: Bob Kiaii, MD FRCSC, London Health Sciences Centre, University Hospital, 339 Windermere Rd, London, Ontario, Canada, N6A 5A5 (E-mail: bob.kiaii@lhsc.on.ca).

J Thorac Cardiovasc Surg 2008;136:702-8 $0022-5223 / \$ 34.00$

Copyright $(2008$ by The American Association for Thoracic Surgery

doi:10.1016/j.jtcvs.2008.02.081
Objective: Traditionally integrated coronary artery revascularization has been described as a 2-stage procedure. We evaluated the safety and feasibility of 1-stage, simultaneous, hybrid, robotically assisted coronary artery bypass grafting surgery and percutaneous coronary intervention.

Methods: Fifty-eight patients underwent simultaneous, integrated coronary artery revascularization in an operating theater equipped with angiographic equipment. Fortyfive patients were men. The mean age was 59 years. All internal thoracic arteries were harvested with robotic assistance. All anastomoses were manually constructed through a small anterior non-rib-spreading incision without cardiopulmonary bypass on the beating heart. Immediately after and within the same operative suite, both angiographic confirmation of graft patency and percutaneous coronary intervention were performed. In 52 patients therapeutic anticoagulation was achieved with the direct thrombin inhibitor bivalirudin.

Results: There were no deaths or wound infections. There was 1 perioperative myocardial infarction. One patient had a stroke, and 3 patients required re-exploration for bleeding. The median lengths of intensive care and hospital stay were 1 and 4 days, respectively. All patients were alive and symptom free at follow-up (mean, 20.2 months; range, 1.1-40.8 months). Long-term angiographic follow-up in 54 patients showed 49 (91\%) patent grafts (mean, 9.0 months; range, 4.3-40.8 months). There were 7 in-stent restenoses and 2 occluded stents.

Conclusion: For multivessel coronary artery disease, simultaneous integrated coronary artery revascularization with bivalirudin is safe and feasible. This approach enables complete multivessel revascularization with decreased surgical trauma and postoperative morbidity. Further studies are necessary to better determine patient selection and long-term outcomes.

$\mathrm{T}$ Treatment options for multivessel coronary artery disease are in a state of flux, with significant advancements in both minimally invasive coronary surgery and percutaneous coronary intervention (PCI). As interventional cardiologists become more aggressive with drug-eluting stent (DES) technology and cardiac surgeons pursue less invasive surgical techniques, the potential complimentary aspects of these 2 revascularization strategies become seemingly more evident. Surgical revascularization confers the survival benefit of the left internal thoracic artery (LITA) to left anterior descending coronary artery (LAD) graft. ${ }^{1}$ Moreover, when faced with significant stenosis of non-LAD coronary vessels, PCI with DESs provides an acceptable alternative to radial artery and saphenous vein grafts, with recognition that follow-up for DESs has only been for a short duration. ${ }^{2-4}$ The disadvantage of PCI 

Abbreviations and Acronyms
$\begin{array}{ll}\text { ACT } & =\text { activated clotting time } \\ \text { ASA } & =\text { acetylsalicylic acid }\end{array}$
BMS = bare-metal stent
CABG = coronary artery bypass grafting
DES = drug-eluting stent
endo $\mathrm{ACAB}=$ endoscopic atraumatic coronary artery bypass
ICR = integrated coronary revascularization
ICS $=$ intercostal space
ICU = intensive care unit
LAD $\quad=$ left anterior descending coronary artery
LITA $\quad=$ left internal thoracic artery
PCI = percutaneous coronary intervention
TIMI = Thrombolysis In Myocardial Infarction

centers on ostial and complex lesions of the LAD, with an increased need for reintervention. ${ }^{5-7}$ Thus far, PCI has been inferior to surgical revascularization when faced with LAD disease and has yet to demonstrate a survival benefit. ${ }^{1,5}$

With this paradox, hybrid or integrated coronary revascularization (ICR) has evolved as an alternative therapy for multivessel coronary artery disease, combining aspects of both the interventional and surgical approaches into a single procedure ${ }^{8}$ ICR combines minimally invasive LITA to LAD revascularization with PCI of the remaining flow, limiting coronary lesions. Integrating the 2 procedures into one therapeutic modality aims to provide the patient with the beneficial aspects of each successive technique in the least invasive way possible.

Still, the theoretic benefits of ICR have largely been overshadowed by logistic challenges inherent to its use and challenges with respect to differing anticoagulation regimens used for surgical coronary revascularization and PCI. Coronary artery bypass grafting (CABG) surgery and PCI necessitate effective coordination of an operating room and a catheterization laboratory, respectively. For this reason, ICR has been largely confined to a 2-staged procedure. Surgical revascularization is typically performed first, followed by PCI of the remaining stenosed arteries several hours, days, or weeks later. ${ }^{9-11}$ Having to routinely accommodate both an operating room and an interventional laboratory has prevented the widespread acceptance of ICR. One solution is to incorporate the 2 techniques into a single simultaneous procedure using an enhanced operating theater with angiographic equipment, ${ }^{12}$ permitting a truly hybrid approach to multivessel coronary artery disease. Although previously conceptualized in the literature, the discordance in anticoagulation protocols for CABG surgery and PCI, together with a lack of infrastructure, ${ }^{13}$ has prevented simultaneous ICR from reaching full fruition. Recently, an enhanced operating theater was constructed at our institution (Figure 1). Moreover, in an effort to develop a unifying anticoagulation regimen suitable to both $\mathrm{CABG}$ and $\mathrm{PCI}$, we evaluated the use of bivalirudin (The Medicines Company, Parsippany, $\mathrm{NJ}$ ) as the sole anticoagulant during simultaneous ICR.

Our group and others have reported independently first case experiences with robotically assisted simultaneous ICR. ${ }^{12,14,15}$ With the hypothesis that ICR performed simultaneously in an enhanced operative suite with bivalirudin would be associated with excellent early and intermediate-term outcomes, we report the experience from our initial case series.

\section{Materials and Methods Patient Population}

Between September 2004 and July 2007, after obtaining ethics approval and each patient's fully informed written consent, 65 consecutive patients underwent ICR. The first 5 patients had ICR as a 2-staged approach because of the learning curve required to familiarize the team with combined procedures in an enhanced hybrid operative suite. The remaining 60 patients underwent simultaneous ICR. All patients had a preoperative computed tomographic scan of the thorax and pulmonary function tests. Acetylsalicylic acid (ASA) was routinely discontinued 7 days before the operation.

Patient history, physical findings, and angiograms were reviewed by all members of the ICR team (the cardiac surgeon, interventional cardiologists, and cardiac anesthesiologists). Selection criteria included patients with double- or triple-vessel disease in whom the LAD lesion was not suitable for PCI but suitable for surgical revascularization and in whom the non-LAD lesions were amenable to PCI. Exclusion criteria consisted of patients with contraindications to robotically assisted endoscopic atraumatic coronary artery bypass (endoACAB) surgery or contraindications to PCI of non-LAD lesions (Table 1).

The sequence and approach to ICR involved initial robotically assisted endoACAB grafting of the LITA graft to the LAD on the beating heart. This was followed immediately by PCI of the non-LAD stenotic coronary artery lesions in the same enhanced hybrid operative suite. Preoperative patient demographics are shown in Table 2.

\section{Surgical Procedure}

The detailed surgical technique of robotically assisted endoACAB has been previously described. ${ }^{12,16}$ Briefly, using robotic assistance the LITA was harvested endoscopically, and a vertical pericardiotomy was performed. The LAD was identified, and the intercostal space (ICS) best suited for direct access was determined. After achieving appropriate anticoagulation (activated clotting time [ACT] >300 seconds), the LITA graft was clipped and cut. Stabilization of the LAD was achieved with the Octopus TE stabilizer (Medtronic, Minneapolis, Minn) inserted through a port in the third ICS. The LITA was manually anastomosed to the LAD on the beating heart through a small non-rib-spreading incision. Intracoronary shunts were used in all patients. LITA graft flow was immediately assessed by using transit-time Doppler scanning (Medi-stem AS, Medtronic).

\section{Anticoagulation Protocol}

In the first 6 patients anticoagulation for the entire procedure was achieved with an intravenous bolus dose of heparin (400 IU/ $\mathrm{kg}$ ). One patient received both an intravenous bolus of heparin 


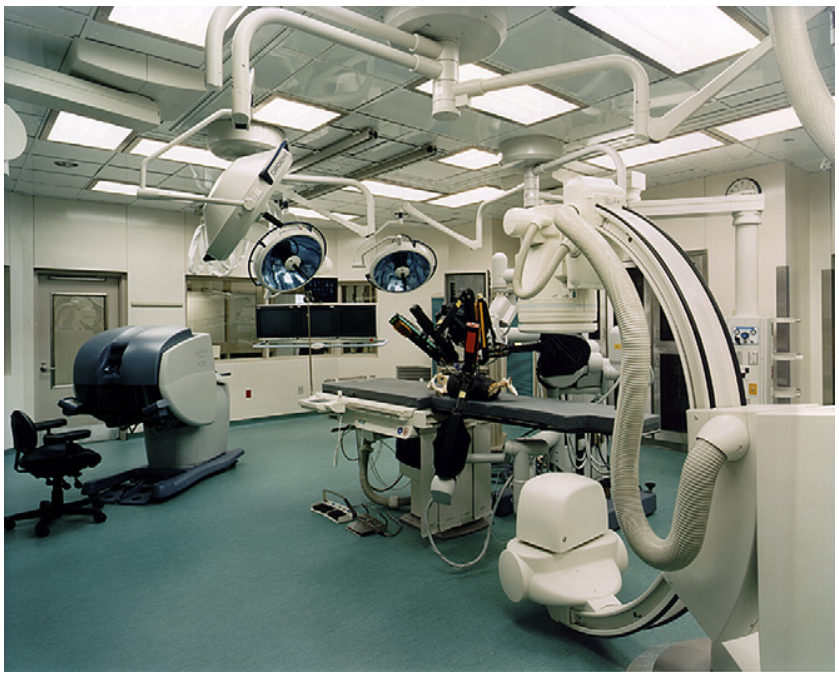

Figure 1. Enhanced operating theater for simultaneous integrated coronary revascularization, depicting the orientation of the carbon fiber operating table, digital C-arm, and daVinci telemanipulation system in relation to one another. Display monitors in the background move to a central position to facilitate angiographic imaging.

(400 IU/kg) for surgical revascularization and bivalirudin at an intravenous bolus of $0.75 \mathrm{mg} / \mathrm{kg}$ followed by an infusion of bivalirudin at $1.75 \mathrm{mg} \cdot \mathrm{kg}^{-1} \cdot \mathrm{h}^{-1}$ for PCI. The remaining 51 patients received bivalirudin alone (intravenous bolus dose of $0.75 \mathrm{mg} / \mathrm{kg}$ followed

TABLE 1. Integrated coronary revascularization inclusion and exclusion criteria

\section{Exclusion criteria}

1. Contraindications to endoACAB

i) Nongraftable LAD

ii) Buried intramyocardial LAD

iii) Inadequate intrathoracic cavity space on thorax CT

iv) Inability to undergo off-pump beating heart

revascularization

v) Previous operation involving the left chest cavity

vi) History of left subclavian artery and LITA stenosis

vii) $\mathrm{FEV}_{1}<50 \%$

viii) $\mathrm{BMI}>40 \mathrm{~kg} / \mathrm{m}^{2}$

2. Contraindications to percutaneous coronary intervention

i) Peripheral vascular disease precluding vascular access

ii) Vessel size $<2.0 \mathrm{~mm}$

iii) Tortuous calcified vessels believed to preclude stent placement

iv) Fresh thrombotic lesion

v) Chronic totally occluded vessels

vi) Serum creatinine $\geq 200 \mu \mathrm{mol} / \mathrm{L}$

endoACAB, Atraumatic coronary artery bypass; $L A D$, left anterior descending; $C T$, computed tomogram; $L I T A$, left internal thoracic artery; $F E V_{1}$, forced expiratory volume in 1 second; $B M I$, body mass index.
TABLE 2. Patient demographics

\begin{tabular}{lc}
\hline \multicolumn{1}{c}{ Profile } & $\mathbf{n}=\mathbf{6 0}(\%)$ \\
\hline Mean age (y) & $59.9 \pm 11.7$ \\
Sex & \\
Male & $47(78 \%)$ \\
Female & $13(22 \%)$ \\
LV grade & \\
I & $45(75 \%)$ \\
II & $12(20 \%)$ \\
III & $3(6.0 \%)$ \\
IV & 0 \\
CCS functional class & \\
I & 0 \\
II & $6(10 \%)$ \\
III & $34(56 \%)$ \\
IVa & $16(27 \%)$ \\
IVb & $3(5 \%)$ \\
IVc & $1(2 \%)$ \\
Recent MI & $10(17 \%)$ \\
Stroke & $2(3.3 \%)$ \\
PVD & 0 \\
COPD & $2(3.3 \%)$ \\
Diabetes & $14(23 \%)$ \\
Preoperative creatinine & \\
$\leq 120$ & $54(90 \%)$ \\
121-180 & $5(8.3 \%)$ \\
BMI $>30$ kg/m ${ }^{2}$ & $1(1.7 \%)$ \\
\hline L Left venticle; & $24(40 \%)$ \\
\hline
\end{tabular}

$L V$, Left ventricle; CCS, Canadian Cardiovascular Society; MI, myocardial infarction; $P V D$, peripheral vascular disease; $C O P D$, chronic obstructive pulmonary disease; $B M I$, body mass index.

by an infusion of $1.75 \mathrm{mg} \cdot \mathrm{kg}^{-1} \cdot \mathrm{h}^{-1}$ ) for the entire hybrid procedure (surgical revascularization and PCI). In all patients who received heparin, this was reversed with protamine sulfate on successful completion of the LITA-LAD anastomosis. For the PCI, an additional heparin bolus was given that was not reversed. In the patients receiving a bivalirudin infusion, the infusion was continued at the same rate of $1.75 \mathrm{mg} \cdot \mathrm{kg}^{-1} \cdot \mathrm{h}^{-1}$ until PCI was completed and the patient was transferred to the intensive care unit (ICU). Throughout the procedure, anticoagulation was monitored by means of frequent ACT measurements to maintain an ACT of greater than 300 seconds. A further half bolus of bivalirudin $(0.375 \mathrm{mg} / \mathrm{kg})$ was administered to enhance the ACT, if necessary.

\section{Percutaneous Intervention}

Immediately after the surgical revascularization, with all incisions closed, the operating room was transformed for PCI. The floormounted $\mathrm{C}$-arm was brought into position, and the display monitors were brought to the center of the operating table. By using femoral access, angiographic confirmation of LITA-LAD patency was verified. PCI of all non-LAD coronary lesions was performed with Taxus (Boston Scientific, Boston, Mass) and Cypher (Johnson \& Johnson, Cincinnati, Ohio) DESs or Cobalt Chromium Driver (Medtronic) bare-metal stents (BMSs). On PCI completion, while still on a bivalirudin infusion, $600 \mathrm{mg}$ of clopidogrel was 
TABLE 3. Technical information for percutaneous coronary intervention

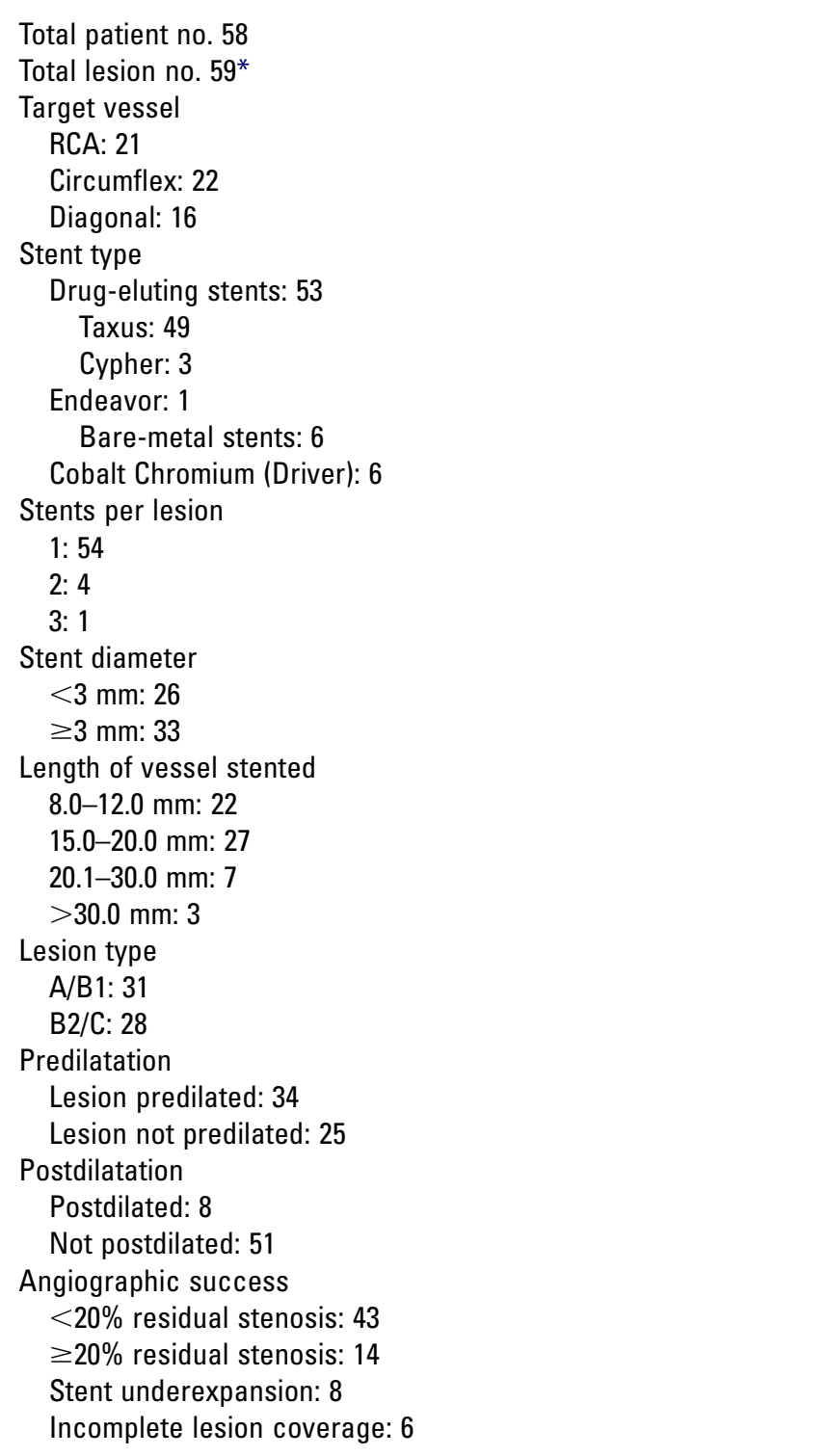

$R C A$, Right coronary artery. ${ }^{*}$ One patient had stenting of both the right coronary and circumflex arteries.

administered through a nasogastric tube, followed by $75 \mathrm{mg}$ of clopidogrel once daily. Eighty-one milligrams of ASA was administered through a nasogastric tube 6 hours after the operation, and then $81 \mathrm{mg}$ was administered once daily. The femoral arterial sheaths were removed 90 minutes after the discontinuation of bivalirudin.

\section{Results}

\section{Intraoperative Results}

Fifty-nine patients had double-vessel and 1 patient had triplevessel coronary artery disease. Of the 60 patients, 58 (97\%) had successful simultaneous ICR. Two patients sustained
TABLE 4. Postoperative events

\begin{tabular}{llr}
\hline \multicolumn{3}{c}{ Average \pm SD Median } \\
\hline Total procedure time (min) & $205 \pm 39$ & 217 \\
Length of stay in ICU (d) & $1.1 \pm 0.43$ & 1 \\
Length of hospital stay (d) & $4.3 \pm 1.42$ & 4 \\
24-h Chest tube drainage (mL) & $809 \pm 724$ & 613 \\
Hemoglobin level at discharge (g/L) & 110 (range, 74-133) & 109 \\
Neurologic complication & 1 patient & - \\
Perioperative myocardial infarction & 1 patient & - \\
Reoperation for bleeding & 3 patients & - \\
\hline
\end{tabular}

ICU, Intensive care unit.

ventricular fibrillation during the beating heart LITA-LAD anastomosis, necessitating defibrillation, which was successful, followed by conversion to sternotomy. Both of these patients underwent complete surgical revascularization offpump through a sternotomy incision by using bivalirudin for anticoagulation and had uncomplicated postoperative courses. Twenty-one patients had PCI to the right coronary artery, 22 patients had PCI to the circumflex artery, and an additional 16 patients had PCI to the first diagonal branch. Detailed technical information of the stenting procedures are provided in Table 3. Intraoperative angiographic analysis confirmed LITA-LAD patency of grade A by means of Fitzgibbons criteria ${ }^{17}$ in 54 (93\%) of 58 patients. In the 4 remaining patients 2 patients had Thrombolysis In Myocardial Infarction (TIMI) flow 1 , and 2 patients had TIMI flow 0 into the LAD. One of the 2 patients with TIMI flow 0 had a chronically occluded LAD preoperatively with very poor collateral flow. Intraoperatively, this patient was found to have a diffusely diseased LAD with a $1.0-\mathrm{mm}$ lumen not very suitable for surgical revascularization. Attempt at revascularization with LITA-LAD anastomoses was made, knowing there would be very poor runoff. Intraoperative angiographic analysis confirmed an occluded anastomosis. The other patient with TIMI flow 0 also had an occluded LAD with collaterals to a small distal LAD segment. A small LAD was confirmed in the operating room. Intraoperative angiographic analysis showed a patent LITA-LAD anastomosis with retrograde flow but no antegrade flow. Because the distal LAD was a small-caliber vessel, the chance of improving this anastomosis was very low; hence this anastomosis was not further revised. Interestingly, this patient had a Fitzgibbons A LITA-LAD anastomosis at long-term follow-up.

\section{Perioperative Results}

Median postoperative length of hospital stay was 4 days (range, 3-9 days). Fifty-five (95\%) patients were transferred to the ward from the ICU within 12 hours. Two patients were in the ICU for 2 days, and 1 patient was in the ICU for 3 days. Re-exploration for bleeding was the cause of the extended ICU stay in all 3 patients (Table 4). A surgical source of 
bleeding was noted in 1 patient, with an arterial bleeder discovered at the camera port site of the fifth ICS. Although initial re-exploration was thoracoscopic, extensive thrombus causing poor visibility necessitated access through the original minithoracotomy, whereby bleeding was then successfully controlled. The other 2 patients were coagulopathic, and a bleeding site was never identified. Because of inadequate exposure and to safely rule out a surgical bleed, after inspection thoracoscopically and directly through the original minithoracotomy, conversion to sternotomy was necessary. Blood transfusions, including packed red blood cells, fresh frozen plasma, and platelets, were required in these 3 patients in addition to 6 other patients.

There were no deaths. One patient had a small postoperative stroke with no clinically significant sequelae. One patient was given a diagnosis of perioperative myocardial infarction evidenced by a new Q-wave in his inferior leads on the postoperative electrocardiogram (Table 4). This individual, who had received heparin and bivalirudin for PCI of the right coronary artery, required re-exploration and conversion to sternotomy for bleeding and received fresh frozen plasma and platelets.

\section{Follow-up}

At a mean follow-up of 20.2 months (range, 1.1-40.8 months), all 58 patients reported Canadian Cardiovascular Society Class 0 angina. Fifty-four (93\%) patients have since returned for long-term angiographic follow-up (mean, 9.0 months; range, 4.3-40.8 months). Two patients were not recatheterized for medical reasons (renal insufficiency and a perioperative stroke), and 2 patients declined recatheterization. The LITA graft was patent in $49(91 \%)$ of 54 patients, and 1 LITA graft was atretic. By using Fitzgibbons angiographic grading criteria, ${ }^{17}$ the LITA was grade A in 36 patients and grade B in 13 patients. Of the patients with a score of grade B, 8 patients had TIMI flow 3 , and 5 patients had TIMI flow 1. One patient with TIMI flow 1 had PCI of the anastomosis with a BMS, even though he was asymptomatic. Another patient with TIMI flow 1, also asymptomatic, had PCI of the native LAD with a DES. One patient's LITA could not be cannulated, and the anastomosis could not be properly visualized to confirm patency. This LITA was very small during intraoperative angiographic analysis and had become atretic by the long-term angiographic assessment because of competitive flow from the collateral circulation to the LAD. In the 5 patients whose LITA grafts were not patent, all were asymptomatic. One patient had the previously discussed chronically occluded LAD with the diffusely diseased vessel (1.0-mm lumen) and TIMI flow 0 at the time of intraoperative angiography. In the 4 remaining patients 2 LITA grafts had evidence of a string sign, 1 had TIMI flow 0 into the LAD, and 1 did not have antegrade flow into the LAD (TIMI flow 0 ) but had prompt retrograde flow. These 4 patients had patent grafts at intraoperative angiographic analysis.
There were 2 occluded stents. In addition, 8 vessels had in-segment stenosis, of which 7 had binary in-segment restenosis and 1 had in-segment stenosis noted on preoperative angiographic assessment and initially judged not to require intervention; however, this vessel was stenosed $50 \%$ or greater at 6-month follow-up. Of the 7 patients with insegment binary restenosis, 2 underwent target lesion revascularization with repeat PCI of the stented vessel using a DES. Of note, 5 of these patients underwent a suboptimal stenting procedure ( $\geq 20 \%$ residual stenosis), and 2 of these patients initially had undergone a BMS insertion (Table 3 ). Of the 53 inserted DESs, 2 were occluded, and 5 had in-segment binary restenosis. The 9 patients with stent complications were all completely asymptomatic and continued to report Canadian Cardiovascular Society Class 0 angina.

\section{Discussion}

The present study demonstrates simultaneous ICR in a single operative sequence for multivessel coronary artery disease to be safe, efficacious, and reproducible. Angelini and colleagues ${ }^{8}$ were the first to describe ICR. This consisted of 6 patients, 4 of whom had a 2-stage procedure and 2 of whom underwent simultaneous ICR with a standard catheterization laboratory. Minimally invasive direct coronary artery bypass surgery was used in Angelini and colleagues' series, whereby the LITA graft was harvested and anastomosed to the LAD directly through an anterior thoracotomy on the beating heart. Minimally invasive direct coronary artery bypass has since fallen out of favor with most surgeons because of the technical challenges of direct LITA harvesting and the morbidity associated with costochondral dislocation and rib fractures. ${ }^{18}$ With the development of computer-enhanced telesurgical systems, minimally invasive surgical coronary artery revascularization has become more feasible. ${ }^{19}$ Moreover, with the introduction of DESs and the potential for lower rates of in-stent restenosis, ${ }^{4}$ ICR might become a more acceptable alternative for revascularization in select patients with multivessel coronary artery disease. To date, the majority of ICR cases have been performed through a 2-stage approach. The few cases that have been performed with robotic assistance or simultaneously in a 1-stage approach have been performed on the arrested heart with cardiopulmonary bypass. ${ }^{14,20,21} \mathrm{We}$ believe the maximal benefit of ICR to be achieved when surgical revascularization is performed with a minimal-access, off-pump, beating heart technique followed by immediate PCI in the same operating theater.

ICR eliminates the need for sternal incisions and the need for cardiopulmonary bypass, while still providing the survival benefit of the LITA-LAD anastomosis and, with the addition of PCI, ensures complete revascularization of all significantly diseased arteries. The avoidance of cardiopulmonary bypass provides certain advantages, mainly reduced ventilation times, ICU and hospital lengths of stay, and 
potential improvements in midterm cognitive function. ${ }^{22}$ In addition, although evidence continues to be conflicting, it has been suggested that beating heart surgery might be associated with lower mortality and major adverse events. ${ }^{23}$ Moreover, simultaneous ICR provides a practical 1-stage procedure that is efficient and convenient for the surgeon, the interventional cardiologist, and, most importantly, the patient. It has been suggested that ICR is best performed as a 2stage procedure and that the use of a single revascularization suite is "suboptimal." 10 It is our contention that the 1-stage approach is a safer and more efficient alternative. With the 2-stage approach to ICR, the patient remains incompletely revascularized and at risk for a cardiovascular event for an extended period of time. Simultaneous ICR reduces this concern. The 1-stage approach also optimizes continuity of care through direct intraoperative collaboration among the surgeon, cardiologist, and cardiac anesthesiologist. Also, with the inevitable increase in hospital length of stay and resource use to coordinate a 2 -staged procedure, simultaneous ICR has the potential to be more cost-effective in the long term. Finally, the effect on patient psyche and satisfaction from having to undergo only 1 procedure as opposed to 2 procedures is likely significant and should not be overlooked.

Simultaneous ICR presents the additional challenge of combining 2 procedures that have different anticoagulation requirements. Overlapping these 2 anticoagulation regimens increases the risk of perioperative hemorrhage. After careful study, bivalirudin was deemed to be an appropriate pharmacologic agent for the entire hybrid procedure. Bivalirudin has been proved both safe and effective as an anticoagulant in patients with unstable angina undergoing PCI. ${ }^{24,25}$ Although there is less experience with bivalirudin in cardiac surgery, recent data suggest bivalirudin to be a clinically feasible alternative to heparin during $\mathrm{CABG}{ }^{26}$

Bivalirudin's ability to inhibit clot-bound thrombin and reduce endothelial proliferation might potentially protect newly sutured grafts in CABG surgery. ${ }^{26}$ In addition, because there is no antidote for bivalirudin and its anticoagulant effects are not reversed abruptly, anticoagulation can persist for some time during the early postoperative phase, providing further graft protection. ${ }^{26}$ Our study supports this idea, whereby the long-term angiographic follow-up in 54 patients showed only 4 of the LITA grafts that were originally patent at intraoperative angiography to have occluded at follow-up. This illustrates a long-term LITA patency rate of $93 \%$, which would indicate the use of bivalirudin to be safe in LITA anastomosis. Thus far, this has been the only long-term angiographic follow-up study of patients undergoing CABG with bivalirudin without cardiopulmonary bypass. ${ }^{27}$ Moreover, the extended period of postoperative anticoagulation appears to provide protection from stent thrombosis until clopidogrel administered immediately after the procedure becomes therapeutic. Only $9(15 \%)$ stents shows complications (7 insegment binary restenoses and 2 occlusions), of which 5 were from the subset of 14 patients having had $20 \%$ or greater residual stenosis at the stent insertion site (Table 3). Excluding these 5 stents with complications caused by technical problems with insertion, only 4 (7\%) stents showed problems with the described anticoagulation and antiplatelet strategy. Although the ideal strategy would be to administer clopidogrel before the procedure, ${ }^{24,25}$ we tried to overlap the effect of bivalirudin and clopidogrel within the 2-hour period after $\mathrm{PCI}^{25}$ by continuing the infusion of bivalirudin after administration of clopidogrel as long as possible until transfer to the ICU. Our goal was to minimize the risk of stent thrombosis with the least amount of postoperative bleeding. Still, 2 patients receiving bivalirudin were found to be coagulopathic and required re-exploration with sternotomy. One patient had received a combination of both intravenous heparin and bivalirudin and the other had thromboelastogram studies verifying ASA-related hyperresponsive platelet dysfunction. ${ }^{28}$ Although speculative, the use of 2 anticoagulants in combination and a hyperresponse to ASA might have contributed to these patient's coagulopathies. After the one case, our protocol was amended; heparin and bivalirudin were no longer used in combination.

Simultaneous ICR is not without potential drawbacks and limitations. Operative times are longer than those of conventional CABG surgery because of the need to coordinate 2 procedures; however, this improves significantly with increased experience. It also necessitates the use of aggressive perioperative antiplatelet therapy, thereby increasing the potential for perioperative blood loss and the need for blood transfusions. Indeed, mean 24-hour chest tube drainage was slightly increased in our series compared with that seen with conventional CABG (Table 4). However, the 3 patients requiring reexploration for bleeding largely account for this increased drainage. Furthermore, only 9 (16\%) patients inevitably required blood transfusions. Also, the inability to tolerate beating heart revascularization and the possible need to convert to sternotomy is always a concern. In this series $4(6.6 \%)$ of 60 patients required sternotomy for bleeding (2 patients) and ventricular arrhythmias ( 2 patients), as previously discussed. Finally, it must be emphasized that the early perceived benefits of DESs have recently come into question, and their long-term safety is still to be determined. ${ }^{29,30}$

In conclusion, robotically assisted simultaneous ICR is a safe, reproducible, and feasible approach to multivessel coronary artery disease in select patient populations. Moreover, bivalirudin is a safe and effective sole anticoagulant for this procedure. Expertise in endoscopic and minimally invasive cardiac surgical techniques, as well as a collaborative, team-oriented approach among cardiac surgeons, interventional cardiologists, and anesthesiologists, is imperative for success.

Randomized prospective studies with long-term patient follow-up comparing simultaneous ICR with conventional CABG procedures or multivessel PCI will be necessary to 
further evaluate the true effect of this technology on patient care and to assess whether this therapeutic approach is associated with similar long-term outcomes.

\section{References}

1. Loop FD, Lytle BW, Cosgrove DM, Stewart RW, Goormastic M, Williams GW, et al. Influence of the internal-mammary graft on 10year survival and other cardiac events. $N$ Engl J Med. 1986;314:1-6.

2. Yun KL, Wu Y, Aharonian V, Mansukhani P, Pfeffer TA, Sintek CF, et al. Randomized trial of endoscopic versus open vein harvest for coronary artery bypass grafting: six-month patency rates. $J$ Thorac Cardiovasc Surg. 2005; 129:496-503.

3. Desai ND, Cohen EA, Naylor CD, Fremes SE. A randomized comparison of radial-artery and saphenous-vein coronary bypass grafts. $N$ Engl $J$ Med. 2004;351:2302-9.

4. Moses JW, Leon MB, Popma JJ, Fitzgerald PJ, Holmes DR, O'Shaughnessy C, et al. Sirolimus-eluting stents versus standard stents in patients with stenosis in a native coronary artery. N Engl J Med.2003; 349:1315-23.

5. Cameron A, Davis KB, Green G, Schaff HV. Coronary bypass surgery with internal-thoracic artery grafts-effects on survival over a 15 -year period. N Engl J Med. 1996;334:216-9.

6. Serruys PW, Unger F, Sousa JE, Jatene A, Bonnier HJ, Schonberger JP, et al. Comparison of coronary-artery bypass surgery and stenting for the treatment of multivessel disease. N Engl J Med. 2001;344:1117-24.

7. Diegeler A, Thiele H, Falk V. Comparison of stenting with minimally invasive bypass surgery for stenosis of the left anterior descending artery. N Engl J Med. 2002;347:561-6.

8. Angelini GD, Wilde P, Salerno TA, Bosco G, Calafiore AM. Integrated left small thoracotomy and angioplasty for multivessel coronary artery revascularization [letter]. Lancet. 1996;347:757-8.

9. Murphy GJ, Bryan AJ, Angelini GD. Hybrid coronary revascularization in the era of drug-eluting stents. Ann Thorac Surg. 2004;78:1861-7.

10. Davidavicius G, Van Praet F, Mansour S, Casselman F, Bartunek J, Degrieck I, et al. Hybrid revascularization strategy: a pilot study on the association of robotically enhanced minimally invasive direct coronary artery bypass surgery and fractional-flow-reserve guided percutaneous coronary intervention. Circulation. 2005;112(suppl):I317-22.

11. Vassiliades TA, Douglas JS, Morris DC, Block PC, Ghazzal Z, Rab ST, et al. Integrated coronary revascularization with drug-eluting stents: immediate and seven-month outcome. J Thorac Cardiovasc Surg. 2006;131:956-62.

12. Kiaii B, McClure RS, Kostuk W, Rayman R, Swinamer S, Dobkowski WB, et al. Concurrent robotic hybrid revascularization using an enhanced operative suite. Chest. 2005;128:4046-8.

13. Rayman R. Robotic-assisted cardiac surgery. Semin Laparosc Surg. 2004;11:73-9.

14. Bonatti J, Schachner T, Bonaros N, Laufer G, Kolbitsch C, Margreiter J, et al. Robotic totally endoscopic coronary artery bypass and catheter based coronary intervention in one operative session. Ann Thorac Surg. 2005;79:2138-41.

15. Srivastava S, Gadasalli S, Tijerina O, Barrera R, Quismundo S, Srivastava V. Planned simultaneous beating-heart totally endoscopic coronary artery bypass (TECAB) and percutaneous intervention in a single operative setting. Innovations. 2006;1:239-42.

16. Boyd WD, Kiaii B, Novick RJ, Rayman R, Ganapathy S, Dobkowski WB, et al. RAVECAB: Improving outcome in off-pump minimal access surgery with robotic assistance and video enhancement. Can J Surg. 2001;44:45-50.

17. Fitzgibbon GB, Burton JR, Leach AJ. Coronary bypass fate: angiographic grading of 14,000 consecutive grafts early after operation and of 1132 after one year. Circulation. 1978;57:1070-4.

18. Calafiore AM, Giammarco GD, Teodori G, Bosco G, D'annunzio E, Barsotti A, et al. Left anterior descending coronary artery grafting via left anterior small thoracotomy without cardiopulmonary bypass. Ann Thorac Surg. 1996;61:1658-63.

19. Argenziano M, Katz M, Bonatti J, Srivastava S, Murphy D, Poirier R, et al. Results of the prospective multicentre trial of robotically assisted totally endoscopic coronary artery bypass grafting. Ann Thorac Surg. 2006;81:1666-74.

20. de Canniere D, Jansen JL, Goldschmidt-Clermont P, Barvais L, Decroly P, Stoupel E. Combination of minimally invasive coronary bypass and percutaneous transluminal coronary angioplasty in the treatment of double-vessel coronary disease: two-year follow-up of a new hybrid procedure compared with "on-pump" double bypass grafting. Am Heart J. 2001;142:563-70.

21. Katz MR, Van Praet F, de Canniere D, Murphy D, Siwek L, SeshadriKreaden U, et al. Integrated coronary revascularization: percutaneous coronary intervention plus robotic totally endoscopic coronary artery bypass. Circulation. 2006;114(suppl):I473-6.

22. Puskas J, Cheng D, Knight J, Angelini G, DeCannier D, Diegeler A, et al. Off-pump versus conventional coronary bypass grafting: a metaanalysis and consensus statement from the 2004 ISMICS consensus conference. Innovations. 2005;1:3-27.

23. Puskas J, Edwards FH, Pappas PA, O’Brien S, Peterson ED, Kilgo P, et al. Off-pump techniques benefit men and women and narrow the disparity in mortality after coronary bypass grafting. Ann Thorac Surg. 2007;84:1447-54

24. Lincoff AM, Bittl JA, Harrington RA, Feit F, Kleiman NS, Jackman JD, et al. Bivalirudin and provisional glycoprotein IIb/IIIa blockade compared with heparin and planned glycoprotein IIb/IIIa blockade during percutaneous coronary intervention: REPLACE-2 randomized trial. JAMA. 2003;289:853-63.

25. Stone GW, McLaurin BT, Cox DA, Bertrand ME, Lincoff AM, Moses JW, et al. Bivalirudin for patients with acute coronary syndromes. N Engl J Med. 2006;355:2203-16.

26. Merry AF, Raudkivi PJ, Middleton NG, McDougall JM, Nand P, Mills BP, et al. Bivalirudin versus heparin and protamine in off-pump coronary artery bypass surgery. Ann Thorac Surg. 2004;77:925-31.

27. Smedira NG, Dyke CM, Koster A, Jurmann M, Bhatia DS, Hu T, et al. Anticoagulation with bivalirudin for off-pump coronary artery bypass grafting: the results of the EVOLUTION-OFF study. J Thorac Cardiovasc Surg. 2006;131:686-92.

28. Agarwal S, Coakley M, Reddy K, Riddell A, Mallett S. Quantifying the effect of antiplatelet therapy: a comparison of the platelet function analyzer (PFA-100) and modified thromboelastography (mTEG) with light transmission platelet aggregometry. Anesthesiology. 2006;105:676-83.

29. Lagerqvist B, James SK, Stenestrand U, Lindback J, Nilsson T, Wallentin L. Long-term outcomes with drug-eluting stents versus bare-metal stents in Sweden. N Engl J Med. 2007;356:1009-19.

30. Rodriguez AE, Maree AO, Mieres J, Berrocal D, Grinfeld L, FernandezPereira C, et al. Late loss of early benefit from drug-eluting stents when compared with bare-metal stents and coronary artery bypass surgery: 3 years follow-up of the ERACI III registry. Eur Heart J. 2007;28: 2118-25. 